\title{
Cadena de abastecimiento Gestión en entornos competitivos
}

Rómulo Edgar Voysest

Rómulo Edgar Vreca

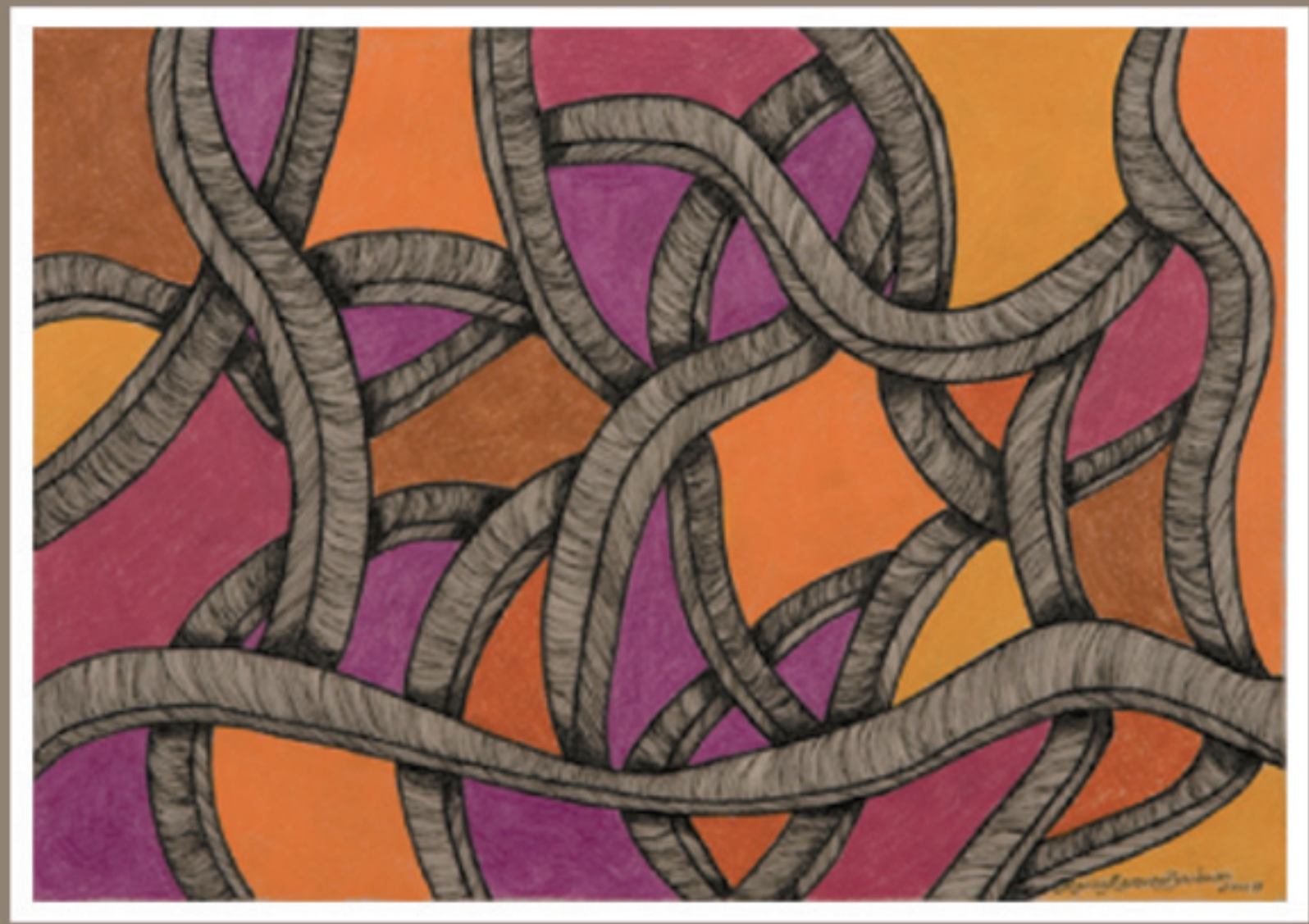

$\boldsymbol{\Theta} \mathrm{UPC}$ 


\section{Cadena de abastecimiento Gestión en entornos competitivos}

Rómulo Edgar Voysest

Rómulo Edgar Vreca 
(C) Universidad Peruana de Ciencias Aplicadas (UPC)

Primera publicación: julio de 2009

Impreso en el Perú - Printed in Peru

Cubierta: Mariza Marques Barbosa

Corrección de estilo: Jorge Coaguila

Diseño de cubierta: Giuliana Abucci / Germán Ruiz Ch.

Diagramación: Diana Patrón

Editor del proyecto editorial

Universidad Peruana de Ciencias Aplicadas S.A.C.

Av. Alonso de Molina 1611, Lima 33, Perú

Telef. 313-3333

http://www.upc.edu.pe

Primera edición: año 2009

Tiraje: 670 ejemplares

Este libro se terminó de imprimir en los talleres gráficos de REP SAC.

Emilio Althaus 355, Lince. Lima, Perú.

\section{Universidad Peruana de Ciencias Aplicadas (UPC) \\ Centro de Información}

Edgar Voysest, Rómulo y Edgar Vreca, Rómulo

Cadena de abastecimiento. Gestión en entornos competitivos

Lima: Universidad Peruana de Ciencias Aplicadas (UPC), 2009

ISBN 978-612-4041-34-1

LOGISTICA, ADMINISTRACION DE VENTAS, DISTRIBUCION COMERCIAL, PLANIFICACION DE LA PRODUCCION, CONTROL DE INVENTARIOS, COMPRAS INDUSTRIALES, ALMACENES

\subsection{EDGA}

Hecho el depósito legal en la Biblioteca Nacional del Perú N²009-06917

Registro de Proyecto Editorial en la Biblioteca Nacional del Perú No 11501400900371

Todos los derechos reservados. Esta publicación no puede ser reproducida, ni en todo ni en parte, ni registrada en o transmitida por un sistema de recuperación de información, en ninguna forma ni por ningún medio, sea mecánico, fotoquímico, electrónico, magnético, electroóptico, por fotocopia o cualquier otro, sin el permiso previo, por escrito, de la editorial.

El contenido de este libro es responsabilidad de los autores y no refleja necesariamente la opinión de los editores. 
Pop art

Serie Cosmos, Mar y Tierra Tinta china y lápiz de color sobre papel $0.30 \times 0.40 \mathrm{~m}$ 2009

La Universidad Peruana de Ciencias Aplicadas (UPC) agradece a Mariza Marques Barbosa de Dianderas la cesión de su cuadro reproducido en la cubierta. 


\section{ÍNDICE}

PRESENTACIÓN

PRÓLOGO

INTRODUCCIÓN

\section{CAPÍTULO 1}

La cadena de suministros (supply chain management) $\quad 33$

I. RESEŃA HISTÓRICA 33

I.1. Sistemas burocráticos $\quad 35$

I.2. Impacto del crecimiento del intercambio comercial 37

I.3. Impacto de las comunicaciones. Del chasqui a internet 38

I.4. Código de barras 38

I.5. Transporte 39

II. DEFINICIÓN DE LA CADENA DE SUMINISTROS 40

II.1. Secuencia de los procesos $\quad 42$

II.2. Tubería de alimentación o pipeline 45

II.3. Objetivos de la cadena de suministros 46

III. INTEGRACIÓN 47

IV. COSTOTOTAL 50

V. PROVEEDORES $\quad 52$

VI. OUTSOURCING O TERCERIZACIÓN 54

VII. ALIANZAS ESTRATÉGICAS 55

VIII. FACTORES GESTANTES DE LA NUEVA FILOSOFÍA 55

IX. SISTEMA DE PRODUCCIÓN TOYOTA (TPS) 57

IX.1. Introducción $\quad 57$

IX.2. De la producción artesanal a la producción en masa $\quad 58$

IX.2.1. Pioneros de la producción en masa $\quad 60$

IX.2.2. Aspectos laborales $\quad 61$ 
IX.3. Desarrollo del sistema de producción Toyota

IX.3.1. Integración vertical versus horizontal 64

IX.3.2. Keiretsu 65

IX.4. Máximo potencial de los trabajadores 66

IX.4.1. Sistema de sugerencias $\quad 66$

IX.4.2. Círculos de calidad $\quad 67$

IX.5. Eliminación del desperdicio $\quad 67$

IX.6. Sistemas push y pull $\quad 71$

IX.7. Reproducir TPS $\quad 72$

X. JUSTO A TIEMPO (JIT) $\quad 74$

$\begin{array}{ll}\text { XI. KANBAN } & 78\end{array}$

$\begin{array}{lr}\text { XII. JIDOKA } & 82\end{array}$

$\begin{array}{ll}\text { XIII. KAIZEN } & 83\end{array}$

XIV. RESUMEN $\quad 85$

XV. LEAN PRODUCTION 86

XV.1. Procesos $\quad 87$

XV.2. Educación $\quad 89$

$\begin{array}{ll}\text { XV.3. Perspectiva } & 90\end{array}$

\section{CAPÍTULO 2}

Planeamiento de ventas $\quad 91$

I. INTRODUCCIÓN 91

II. MODELO DE PLANEAMIENTO 92

III. PRONÓSTICO DE VENTAS 95

III.1. Estrategias de producción e inventarios $\quad 95$

III.2. Fuentes de información $\quad 97$

$\begin{array}{ll}\text { III.2.1. Factores cuantitativos } & 97\end{array}$

III.2.2. Factores cualitativos 98

IV. ANÁLISIS DE LA ESTADÍSTICA DE VENTAS 99

IV.1. Demanda histórica 99

IV.2. Variabilidad 99

IV.3. Tendencia 101 
IV.4. Demanda estacional o estacionalidad

V. MÉTODOS DE PRONÓSTICO DE VENTAS 106

VI. PROMEDIOS MÓVILES

VII. ANÁLISIS DE REGRESIÓN

IX. REVISIÓN Y APROBACIÓN DEL PLAN DE VENTAS 118

X. CICLO DE PLANEAMIENTO,

LEAD TIMES E INVENTARIOS

X.1. Ciclo de planeamiento

X.2. Lead times

X.3. Inventarios

XI. INDICADORES

\section{CAPÍTULO 3}

Planeamiento de la producción

I. INTRODUCCIÓN

II. COORDINACIÓN DE VENTAS, PRODUCCIÓN Y SUMINISTROS

III. PROGRAMA DE PRODUCCIÓN

(MASTER PRODUCTION SCHEDULE)

III. 1. Diversidad y clasificación de productos

III. 2. Prioridades de ventas

III. 3. Cuellos de botella o restricciones de producción

III. 4. Distribución

IV. UN EJEMPLO DE PROGRAMACIÓN DE LA PRODUCCIÓN 132

IV.1. Modelos de planeamiento de la producción

V. REVISIÓN DEL PROGRAMA DE PRODUCCIÓN

VI. MRP Y MRP II

VII. LEAN PRODUCTION

VII.1. Producción impulsada por la demanda 
VII.2. Cambio del paradigma de la producción en masa

VII.3. Estrategia para reducir costos

VII.4. Automatización y diseño

VII.5. Individualizar (customize)

VII.6. Punto de desacople

VII.7. Requisitos para implantar lean production

VII.8. Conclusión

VIII. EL CICLO DE VIDA DE UN PRODUCTO

IX. EL CICLO DE VIDA DE LOS PROCESOS

X. LA CURVA DE APRENDIZAJE

XI. CONCLUSIÓN

XII. INDICADORES

\section{CAPÍTULO 4}

\section{Planeamiento estratégico de la red de suministros}

I. INTRODUCCIÓN

II. TIPOS DE DEMANDA 153

II.1. Independiente 153

II.2. Dependiente 153

III. CLASES DE MATERIALES 153

III.1. Directos 154

III.2. Para mantenimiento, reparación y operaciones (MRO) 154 III.2.1. Incertidumbre de los materiales MRO 155

IV. SISTEMAS DE PLANEAMIENTO Y CONTROL DE MATERIALES

V. PLANEAMIENTO DE REQUERIMIENTOS DE MATERIALES (MRP)

V.1. Objetivos

V.2. Inputs para el sistema MRP

V.2.1. Lista de materiales (BOM) 161

V.3. Outputs del sistema MRP

V.3.1. Explosión de materiales 
V.4. Ejemplo simplificado de MRP

V.5. Resumen

VI. MRP II

VII. CANTIDAD ECONÓMICA DE COMPRA O

CANTIDAD FIJA PERIODO VARIABLE (EOQ)

VII.1. Introducción

VII.2. Determinación de los parámetros de costos $\quad 170$

VII.3. Costo de posesión

VII.4. Costo de adquisición

VII.5. Costo de quiebre de inventario

VII.6. Cálculo de la cantidad económica o lote económico (EOQ) 173 VII.6.1. Condición de certidumbre

VII.6.2. Condición de incertidumbre

VII.7. Variación del precio

VII.8. Inventario de seguridad

VII.9. Grado de servicio

VII.9.1. Uso de la tabla de valores z

VII.10. Punto de pedido

VII.11. Resumen del modelo EOQ

VIII. PERIODO FIJO, CANTIDAD VARIABLE

IX. PERIODO DE REPOSICIÓN (LEAD TIME)

IX.1. Actividades del periodo de reposición

IX.2. Tiempo de reposición

IX.3. Variación del lead time y su efecto en el suministro

204

X. CONCEPTOS ADICIONALES

204

XI. MODELOS MATEMÁTICOS

205

XII. LABOR DEL ANALISTA

XII.1. Situaciones de emergencia

206

XIII. SELECCIÓN DEL SISTEMA DE PLANEAMIENTO Y CONTROL

XIV. FACTORES CRÍTICOS DE ÉXITO 
XIV.2. Actitud proactiva

XIV.3. Cualidades personales 210

XIV.4. Seguimiento 211

XIV.5. Mantener el nivel óptimo de los inventarios 211

XIV.6. Solución de contingencias 211

\section{CAPÍTULO 5}

Inventarios 213

I. INTRODUCCIÓN 213

I.1. Dinámica de la cadena de suministros 214

I.2. Inventarios redundantes 216

I.3. ¿Cuándo se inicia el inventario? 217

I.4. Los inventarios como fuente de ingreso 218

II. FUNCIÓN DE LOS INVENTARIOS 219

II.1. Inventario corriente o cíclico 219

II.2. Inventario de seguridad 219

II.3. Inventario en proceso 219

II.4. Casos especiales 220

II.4.1. Productos de temporada 220

II.4.2. Compras especulativas 220

II.4.3. Productos de bajo volumen de ventas 220

III. COSTO DE LOS INVENTARIOS 221

IV. COSTO DE POSESIÓN 221

IV.1. Introducción 221

IV.2. Componentes del costo de posesión 222

IV.3. Casos 226

IV.4. Estimado del costo de posesión 227

V. COSTO DE ADQUISICIÓN 228

VI. ÁMBITO DE LOS INVENTARIOS 229

V.1. Conflicto de intereses 229

V.2. Esfuerzo común 230

V.3. Casos especiales 230 
VII. CONTROL DE INVENTARIOS

VIII. LA LEY DE PARETO 232

VIII.1. Valor crítico 235

VIII.2. Otras aplicaciones 236

IX. ROTACIÓN DE INVENTARIOS 236

X. MATERIALES SIN MOVIMIENTO, EXCEDENTES Y OBSOLETOS 238

X.1. Disposición de los materiales excedentes, obsoletos y deteriorados 239

XI. POLÍTICA DE INVENTARIOS 241

XI.1. Importancia de una política de inventarios 241

XI.2. Política de inventarios-productos terminados 242

XI.3. Política de inventarios-materiales directos 243

XI.3.1. Posición aprobada de inventarios $\quad 245$

XI.3.1.1. Inventario disponible 247

XII.3.1.2. Inventario en tránsito 250

XII.3.1.3. Pedidos en proceso 251

XII.3.1.4. Posición aprobada total 252

XI.3.2. Comentario final 253

XI.3.3. Casos prácticos 254

XI.3.4. Inventario de seguridad 258

XI.3.5. Producción impulsada por la demanda

(demand driven production) 259

XI.3.6. Tiempo de desembolso a ingreso 260

XI.3.7. Globalización 260

XI.3.8. El efecto látigo de arriero (bullwhip) 261

XI.4. Política de inventarios, suministros MRO 261

XI.4.1. Clasificación de los materiales MRO 262

XI.4.2. Modo de consumo de los materiales MRO 263

XI.4.2.1. Piezas de desgaste 263

XI.4.2.2. Piezas de consumo aleatorio 264 
XI.4.2.3. Repuestos estándar

XI.4.2.4. Suministros varios 265

XI.4.3. Control de inventario de suministros MRO 265

XI.4.3.1. Proveedor integrado 266

XI.4.3.2. Rotación $\quad 267$

XI.4.3.3. Base de datos $\quad 267$

XI.4.4. Nuevas técnicas de gestión de materiales 268

XI.4.4.1. Sistema inalámbrico 268

XI.4.4.2. Sistema integrado 268

XI.4.5. ¿Por qué crear inventarios MRO? 268

XI.4.5.1. Disponibilidad 268

XI.4.5.2. Valor 269

XI.4.5.3. Repuestos críticos 269

XI.4.5.4. Frecuencia de uso $\quad 269$

$\begin{array}{ll}\text { XII. ESTANDARIZACIÓN } & 271\end{array}$

XII.1. Especificaciones técnicas $\quad 271$

XII.1.1. Información y entrenamiento $\quad 272$

XII.1.2. ¿En qué consisten las especificaciones técnicas? 273

XII.1.3. Estandarizar por marca 274

XII.1.4. Nomenclatura y base de datos 275

XII.1.5. Catálogo de repuestos MRO 276

$\begin{array}{ll}\text { XIII. CONCLUSIÓN } & 277\end{array}$

\section{CAPÍTULO 6}

$\begin{array}{ll}\text { Compras } & 279\end{array}$

I. INTRODUCCIÓN 279

II. EVOLUCIÓN DEL ÁREA DE COMPRAS 280

II.1. De integración vertical a integración horizontal 281

II.2. Automatización 282

II.3. e-Procurement 283

II.3.1. Tarjetas de compra 284 
III. OPERACIONES DE COMPRA 285

IV. CALIDAD 286

IV.1. Desarrollo de nuevos productos 287

IV.2. Educación y entrenamiento 288

IV.3. La nueva perspectiva de calidad 289

V. ENTREGA 290

V.1. Comentarios sobre la cantidad a pedir 290

V.2. Problema de entrega por falta de disponibilidad 291

V.3. Seguimiento 292

VI. COSTO 295

VI.1. El precio justo $\quad 296$

VI.2. La oferta y la demanda 298

VI.3. La competencia 299

VI.4. El poder de negociación $\quad 300$

VI.5. El presupuesto anual 301

VII. COTIZACIONES COMPETITIVAS 302

VIII. NEGOCIACIÓN 304

VIII.1. Planeamiento y preparación para la negociación 305

VIII.2. Información 306

VIII.3. Organización 308

VIII.4. Técnicas de negociación 308

IX. ANÁLISIS DE COSTOS 310

IX.1. Análisis costo-volumen $\quad 312$

IX.2. Punto de equilibrio $\quad 314$

IX.3. ¿Comprar o fabricar? $\quad 315$

X. SERVICIO 317

XI. RESPONSABILIDADES COLATERALES 319

XI.1. Compras como centro de utilidades 319

XI.2. Reducción de inventarios 320

XI.3. Imagen de la empresa 321

XI.4. Inteligencia de mercado 321 
XII. LA ORGANIZACIÓN DE COMPRAS

XII.1. Relaciones internas

XII.2. Organigrama

325

XIII. TAREAS ADMINISTRATIVAS DE COMPRAS

326

XIII.1. Estadística

326

XIII.2. Biblioteca de catálogos

XIII.3. Archivos

XIII.4. Mantenimiento de la base de datos

XIV. ANÁLISIS DE VALOR (VALUE ANALYSIS)

XIV.1. Historia

XIV.2. Metodología

XV. DESARROLLO DE PROVEEDORES

$\mathrm{XV}$.1. Ubicar nuevos proveedores

$\mathrm{XV}$.2. Mejorar proveedores existentes

XVI. ASPECTOS LEGALES DE COMPRAS

XVI.1. Contratos

XVII. COMERCIO INTERNACIONAL

XVII.1. Tarifas e impuestos de importación

XVII.2 Incoterms

XVII.3. Transporte multimodal

XVIII. CÓDIGO DE ÉTICA

XVIII.1. Control

XIX. FACTORES CRÍTICOS DE ÉXITO 350

XIX.1. Reducción de costos 352

XX. MEDICIÓN DEL DESEMPEÑO 352

\section{CAPÍTULO 7}

\section{Almacenes}

I. INTRODUCCIÓN

II. FUNCIONES DEL ALMACÉN 356

III. RECIBIR 357

III.1. Aviso anticipado de embarque $\quad 357$

III.2. Documentos de embarque 358 
III.3. Inspección y control de calidad en recepción

III.4. Daños y pérdidas en tránsito

IV. ALMACENAR

360

IV.1. Reglas básicas

360

IV.2. Exactitud de los inventarios

362

IV.3. Las $5 \mathrm{~s}$

364

V. DESPACHAR

365

VI. DISEÑO Y EQUIPAMIENTO DEL ALMACÉN

366

VI.1. Andamios y similares

367

VI.2. Contenedores

369

VI.3. Equipo móvil

370

VI.3.1. Capacidad de carga y altura

370

VI.3.2. Tipo de llantas

370

VI.3.3. Ancho de pasadizos

370

VI.3.4. Fuente de energía

372

VI.3.5. Accesorios (opciones)

372

VI.3.6. Estabilidad

373

VI.3.7. Estabilidad lateral

374

VI.3.8. Estabilidad longitudinal $\quad 375$

VI.3.9. Estabilidad dinámica

VI.3.10. Seguridad en la operación de los montacargas $\quad 378$

VII. SISTEMA DE INFORMACIÓN DEL ALMACÉN

VIII. FACTORES CRÍTICOS DE ÉXITO

IX. INDICADORES

\section{CAPÍTULO 8}

\section{Distribución física}

I. INTRODUCCIÓN 383

II. CANALES DE DISTRIBUCIÓN 384

III. DISTRIBUCIÓN FÍSICA INTERNACIONAL 386

IV. GESTIÓN DEL ALMACÉN DE PRODUCTOS TERMINADOS 
IV.1. Almacenar

IV.2. Ubicación del almacén de productos terminados o centro de distribución

IV.3. Servicios contratados, 3PL (outsourcing)

IV.4. Despachar

IV.4.1. Picking

V. TRANSPORTE

V.1. Ruteo de vehículos

V.2. Guía de transporte

VI. SISTEMA DE INFORMACIÓN

VII. SERVICIO AL CLIENTE

VIII. COLABORACIÓN INTERNA

IX. COSTOS DE DISTRIBUCIÓN

X. FACTORES CRÍTICOS DE ÉXITO

BIBLIOGRAFÍA 


\section{Presentación}

El cambio vertiginoso al que se enfrenta el mercado en todas sus áreas ha provocado que las empresas cuestionen su forma actual de hacer negocios. La velocidad de estos cambios y el éxito de algunas empresas para adaptarse a los mismos han hecho que las antiguas prácticas de gestión, que en su tiempo posibilitaron el éxito, ya no sean válidas. Para alcanzar el éxito se debe desarrollar una estrategia que genere valor al producto o servicio que se ofrece, puesto que si éste no se encuentra disponible para el cliente en el momento y el lugar que ellos desean consumirlo, carecerá de cualquier beneficio.

Para crear dicho valor, la empresa debe enfocarse en cuatro puntos medulares: forma, tiempo, lugar y propiedad. El primero es una función natural del área de producción. El segundo y el tercero son responsabilidades directas de la logística. En este sentido, como tres de los cuatro criterios de valor pertenecen a la cadena de abastecimiento, ésta crece en dimensión e importancia dentro de la empresa como factor determinante para alcanzar el valor esperado en el producto o servicio. En este enfoque, las compañías líderes serán aquellas que sean capaces de tener éxito en el diseño de su cadena de abastecimiento.

Es así que aparece este libro, Cadena de Abastecimiento. Gestión en entornos competitivos, que a través de sus páginas lleva al lector por este fascinante mundo de la cadena de abastecimiento. El libro posee una estructura adecuada para el entendimiento de las variables y los factores que deben ser tomados en consideración cuando se diseña una cadena de abastecimiento y, sobre todo, las claves para competir en mercados cada vez más competitivos. Aborda siete 
puntos clave en la elaboración de la cadena, como son el planeamiento, las ventas, la producción, los inventarios, las compras, los almacenes y la distribución, y centra su atención en la interacción e integración para afrontar el desafío de ser buenos y con calidad en mercados competitivos.

Es por ello que siento un gran placer y es para mí un honor que me hayan encomendado el prólogo de esta publicación, que estoy seguro aportará, no solo en el campo de la ingeniería si no también en el de la organización, una visión de gestión integral logística: gestión de la cadena de abastecimiento.

\section{Gustavo Guerrero}

Decano de la Facultad de Ingeniería de la Universidad Peruana de Ciencias Aplicadas (UPC) 


\section{Prólogo}

Las especialidades en la administración de negocios nacen de las necesidades creadas por la evolución y el crecimiento de los negocios, apoyados por el desarrollo de la tecnología. La cadena de suministros (supply chain), por ejemplo, es producto de la combinación de una serie de factores que se pueden sintetizar en la necesidad de crear las condiciones para mejorar la competitividad de una empresa. Dichos factores pueden ser mejorar la calidad, reducir costos, optimizar el servicio al cliente, evitar desperdicios, etcétera. Al mismo tiempo, la gestión moderna de la cadena de suministros es factible gracias a la nueva tecnología de información y comunicaciones, sin cuyas facilidades estaríamos estancados en sistemas burocráticos, lentos, ineficientes y, lo que es peor, sin posibilidad de integrarnos con nuestros pares.

Podría pensarse a simple vista que los problemas de mejorar la competitividad, reducir costos, optimizar la calidad no tienen nada que ver con funciones logísticas (la cadena de suministros), pero sí tienen que ver y mucho.

En un negocio unipersonal el dueño es todo: es obrero y gerente. El pequeño empresario, por lo general, es experto en una determinada especialidad: puede ser un artesano o un profesional en ingeniería u otras carreras de alta tecnología, y se las arregla para realizar tareas administrativas y de ventas. Pero, a medida que el negocio crece, surge la obligación de mantener una contabilidad en orden y pagar impuestos, se precisa ayuda especializada en producción, ventas y suministros. El empresario ya no puede hacer todo él solo, y debe concentrarse en lo que mejor sabe hacer y contratar especialistas en las otras ramas del negocio, los faculta en sus respectivas especialidades y crea una organización. Así, las 
diferentes especialidades pueden interactuar y producir mejores resultados que si lo harían aisladamente.

Ignorar las especialidades puede producir efectos negativos. Algunas empresas, por ejemplo, subordinan las funciones de compras al departamento de administración y finanzas; los almacenes, a producción; y distribución física a ventas. Esta dispersión de funciones es un desperdicio porque requiere especialistas logísticos (almacenes, control de inventarios y compras) en tres áreas diferentes, causando problemas de coordinación y comunicación.

Uno de los principales activos de la empresa son los inventarios. Una empresa con una sólida organización, liderazgo, y un buen sistema logístico se caracteriza por tener un buen control de los inventarios. El control de los inventarios se inicia con el planeamiento de ventas y producción, que es el primer paso del ciclo de negocios. Si no se dan estas condiciones, se abona el terreno para la improvisación, faltantes de materia prima o repuestos vitales para el mantenimiento de maquinaria, pérdida de producción y deficiente atención a los clientes. En otros casos, las deficiencias se reflejan en el inventario en forma de excedentes, obsolescencias y, en los casos más serios, deterioro de productos que terminan en la zona de chatarra. Estas son pérdidas reales, cuyas causas muchas veces no son detectadas por los más altos directivos de la empresa y cuando suena la señal de alarma en los estados financieros se busca culpables, en lugar de analizar las causas del problema.

Desarrollar y producir un artículo y hacerlo llegar a los consumidores involucra no solo procesos de producción y ventas, sino también servicios logísticos, sin los cuales el ciclo de negocios estaría incompleto.

Las actividades logísticas apoyan a las demás actividades del negocio y, de alguna manera, sirven de puente entre los diferentes procesos operativos. Una eficiente función logística permite a los gerentes de producción y de ventas concentrarse en el núcleo de su función y no distraer valiosas energías en especialidades que no le son afines, y dejar estas en manos de especialistas.

Hay que distinguir entre la letra y el espíritu de los procedimientos. Esto último radica en el análisis e interpretación de los datos, la actitud frente a los problemas que se presentan durante el curso de los procesos de suministros, y 
las decisiones gerenciales. La cadena de suministros constituye una actividad de servicio, de héroes anónimos, porque nunca se ganan glorias, pero sí se reciben críticas por la mínima falla. El personal de suministros debe ser proactivo y aprender a convivir con situaciones extremas. Ciencia e ideología se unen como cuerpo y alma para producir los mejores resultados.

Desafortunadamente, hay ignorancia respecto a la cadena de suministros en nuestro medio, un problema que podría explicarse por el tamaño de la mayoría de las empresas nacionales que no permite costear un departamento de suministros. Sin embargo, esta situación está siendo superada y en la actualidad hay no solo mayor difusión, sino formación profesional de ejecutivos en todas las áreas de la cadena de suministros.

Acertadamente se ha dicho que el ejecutivo de suministros no tiene que ser un experto en una ciencia en particular, pero debe saber un poco de muchas cosas, saber aplicarlas, y, sobre todo, poseer una sobredosis de sentido común.

General Motors, Southern Perú Copper Corporation y Goodyear del Perú han aplicado en el Perú sistemas de suministros integrados que pueden servir como modelo para otras empresas.

La experiencia ganada durante muchos años de vida profesional y, sobre todo, la comprobación de la necesidad de educación formal han sido los incentivos para escribir este libro como una contribución a las nuevas generaciones y personas interesadas en esta importante rama profesional. Los conocimientos y la experiencia acumulados durante todos estos ańos no deben perderse, sino que deben servir a los gerentes de suministros de la nueva generación. La evolución científica ha hecho obsoletos los procedimientos manuales, como la desaparición del Kardex manual, el papeleo, el procesamiento de datos en batch, los registros tipo ledger, el cierre manual de libros, las comunicaciones internas con memorandos entregados con cargo por mensajeros, etcétera. En la actualidad, aun en las empresas más pequeñas, hay algún tipo de programa computarizado para registrar y procesar la información que antiguamente se hacía con gran consumo de mano de obra. Los principios básicos siguen tan vigentes como la ley de la gravedad. Pero además se han agregado conceptos, como la eliminación de desperdicios, flujos continuos, calidad total, etcétera, y, 


\section{INTRODUCCIÓN}

\section{Historia}

La función logística es tan antigua como la humanidad, aunque en la estructura organizacional de las empresas modernas es relativamente nueva. La función logística se percibía como un servicio al área de manufactura a la cual generalmente estaba subordinada. La aparición de nuevos y más agresivos competidores, la globalización y las nuevas tecnologías han creado la necesidad de profesionales logísticos capaces de manejar el desarrollo de nuevos y complicados productos, junto con la necesidad de investigar nuevos mercados y reducir costos, etcétera. A partir de esta tecnificación de las operaciones logísticas se ha despertado la atención como un proceso paralelo a producción, ventas y finanzas, que se ha dado en denominar cadena de suministros por la forma como se enlazan sus actividades a lo largo de todo el proceso de negocios. Suministros sigue siendo una función de servicio y apoyo, principalmente a producción y ventas, pero dentro de un marco muy técnico y sofisticado. Su relación con finanzas es respecto al control de los inventarios y los medios de pago a los proveedores de materiales y servicios.

En muchas empresas, sin embargo, aún existen dudas sobre el rol de la gestión de la cadena de suministros. Algunas optan por dividirla en secciones como compras, almacenes y distribución física subordinadas a otras funciones. Las empresas más desarrolladas hacen lo opuesto, es decir, integrar las actividades logísticas y asignarle un rol importante al mismo nivel que ventas, producción 
y finanzas. La integración de las actividades logísticas es lo que conocemos actualmente como la cadena de suministros.

El nombre 'logística' proviene de la institución militar, donde es soporte indispensable. Es una función que decide la victoria o la derrota. En la historia hay innumerables ejemplos de batallas que se han ganado debido a un eficiente servicio logístico. Dos ejemplos recientes son la Guerra del Golfo y el despliegue que hizo Estados Unidos de material bélico y vituallas en la llamada Guerra contra el Terrorismo en Afganistán. La verdad es que ni el más genial de los generales podría ganar una guerra si no dispone de los recursos necesarios en el lugar y en el momento requeridos. La perfección del sistema logístico en la Guerra del Golfo mereció en su momento un suplemento especial de la revista Modern Materials Handling.

La cadena de suministros es el concepto más moderno y generalmente aceptado. En el transcurso del tiempo las empresas han usado denominaciones diferentes, como logística, suministros, o gestión de materiales, aparte de aquellas cuyas funciones logísticas no están integradas en una unidad organizativa, como indicamos más arriba. Últimamente se ha dado en llamar logística a las actividades de transporte, en particular las relacionadas con la distribución física. Por este motivo, pensamos que la estandarización del concepto de la cadena de suministros es lo más apropiado.

\section{La logística en el Perú}

En el Perú hay empresas nacionales y extranjeras que son modelos de buena organización logística. Lamentablemente todavía existen personas que creen que su «experiencia» en actividades domésticas los califica como expertos en logística industrial. Un director de un grupo corporativo muy grande quería eliminar al único almacenero de productos químicos en una de las fábricas del grupo. Su justificación: «ipara qué quieres almacenero si yo en mi chacra guardo las cosas en un cuarto con un candado?». Es triste comprobar que este grado de ignorancia da paso a la arrogancia y al capricho.

No extraña que en esa misma empresa (fábrica de cajas de cartón) la cadena de suministros era un caos. Sin una política definida de suministros y 
sin liderazgo, había tremendos conflictos entre producción, ventas y logística. El pronóstico de ventas era propiedad exclusiva del departamento de ventas, sin ninguna coordinación con las otras áreas. Se comprometía la venta de cajas en cantidades pequeñas no rentables, se cambiaba continuamente el programa de producción y se incumplían las fechas de entrega prometidas. Por razones técnicas no era posible producir exactamente las pequeñas cantidades requeridas por muchos clientes, resultando saldos de 10 a 20 por ciento que pasaban a inventario y después de poco tiempo se convertían en invendibles. Cada cierto tiempo el almacén se veía obligado a eliminar toneladas de productos como desperdicio y aparecía como culpable por «el mal manejo de inventario y el deterioro de los productos». Es solamente lógico que si un producto no se vende, el inventario se acumula, y todo producto en inventario tiende a deteriorarse. Por ejemplo, el cartón corrugado es higroscópico y al absorber la humedad del ambiente pierde resistencia y no es apto para la venta. Una de las características que un gerente de suministros cuida al adquirir materiales es el tiempo de vida en almacenaje.

La desafortunada experiencia que relatamos en el párrafo anterior es real y, por supuesto, un ejemplo de lo que no se debe hacer con una organización. Algunos gerentes escuchan términos como calidad total, justo a tiempo, reingeniería, etcétera, y asumen que son prácticas que se pueden hacer de la noche a la mañana con solo decir vamos a hacer tal o cual proyecto, sin tener los conocimientos profundos, entrenamiento, e infraestructura requeridos ni menos aún estar comprometidos y sin comprender que calidad total no es un proyecto, sino una forma de vida, una actitud mental que debe ser desarrollada. Y piensan que cortar cabezas es, por ejemplo, una forma de reingeniería.

\section{La logística moderna}

La logística moderna es integral. Se basa en el nuevo concepto de la cadena de suministros, dos de cuyos principios fundamentales, además de la integración, son la comunicación y colaboración entre las diferentes áreas.

Logística es una ciencia y un arte. Como ciencia, usa conocimientos de las ciencias matemáticas, física, química, derecho y economía. Se ha dicho 
De manera similar, la cadena de suministros es un proceso compuesto por una serie de subprocesos, durante los cuales se agrega valor al producto mediante el suministro de insumos o la realización de algún servicio logístico, hasta completar un producto final que se entrega al cliente o consumidor. Véase el diagrama que sigue a continuación:

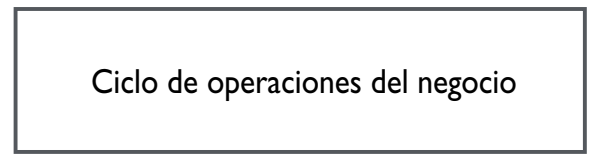

Actividades de la cadena de suministros

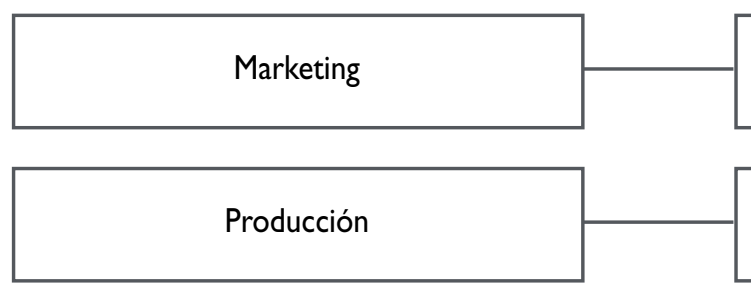

Plan de ventas

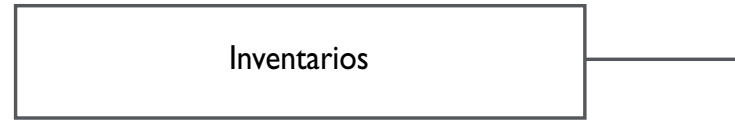

Plan de producción; requerimientos de materiales; compras

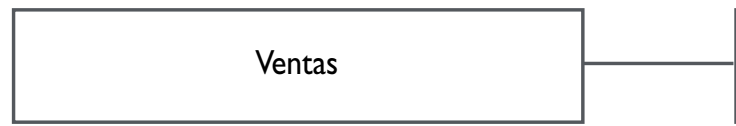

Disponibilidad de productos terminados; gestión de pedidos

Control de inventarios: materia prima; MRO; productos terminados

Distribución física

Almacén; despacho; transporte; entrega

\section{EL CICLO DE NEGOCIOS Y LA CADENA DE SUMINISTROS}

Una peculiaridad de la gestión de la cadena de suministros es que el trabajo tiene que ser "perfecto». Se admite que un grado de perfección al 100 por ciento es imposible, más aún considerando la cantidad de variables y las contingencias que se presentan, pero en la práctica nuestros clientes no nos conceden un margen de tolerancia. La paralización de una máquina por falta de un repuesto, o la pérdida de producción por falta de algún material o un problema con un cliente por un mal despacho son ocurrencias demasiado dramáticas para intentar siquiera una 
explicación. No importa quién sea el culpable, el dedo acusador siempre apunta a logística. Si se hacen 100 cosas bien y una mal, todo está mal. Si se asume esta condición como un reto, y no como un castigo, puede ser una ocupación que produzca la gran satisfacción de la labor cumplida.

El primer capítulo de este libro trata sobre la cadena de suministros. Los principios en que se basa son de suma importancia y cualquier empresa los puede adoptar con algunas variaciones.

En los siguientes capítulos se discuten los sistemas que conforman la cadena de suministros, tratando de mantener una secuencia lógica tal como sucede en la práctica. Un ciclo de negocios se inicia con un plan de ventas y le siguen los planes de producción, planeamiento estratégico de la red de suministro, inventarios, compras, almacenes y distribución física. Cada uno de estos temas ocupa un capítulo del libro.

A lo largo de todo el libro, se hace hincapié en el estado de alerta y actitud proactiva de los directivos de logística como una condición esencial en el desempeño de su trabajo, y se han incluido algunas anécdotas para ilustrar las vivencias en el ejercicio de la profesión.

Los estilos y métodos de gerencia, incluyendo los modernos métodos de medición del rendimiento, no ayudan mucho, si no están respaldados por una conciencia de calidad total, liderazgo, y la voluntad de realizar un trabajo científico en forma sistemática. 
Para poder revisar todo el contenido de esta edición, visite nuestra tienda virtual.

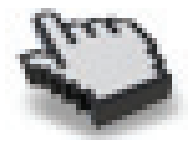

Cadena de abastecimiento

Gestión en entornos competitivos

Rómulo Edgar Voysest Rómulo Edgar Vreca

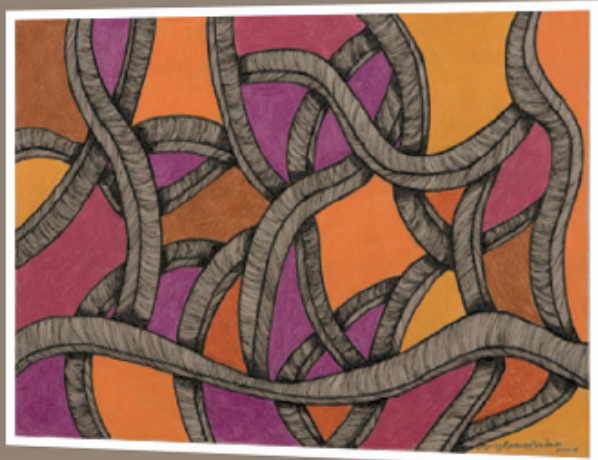

U UPC 